\title{
MRI Texture Analysis of Background Parenchymal Enhancement of the Breast
}

\author{
Yasuo Amano, ${ }^{1}$ Jun Woo, ${ }^{1}$ Maki Amano, ${ }^{2}$ Fumi Yanagisawa, \\ Hiroshi Yamamoto, ${ }^{3}$ and Mayumi Tani ${ }^{4}$ \\ ${ }^{1}$ Department of Radiology, Nihon University Hospital, 1-6 Kanda-Surugadai, Chiyoda-ku, Tokyo 101-8309, Japan \\ ${ }^{2}$ Department of Radiology, Juntendo Nerima Hospital, 3-1-10 Nerima-ku, Tokyo 177-8521, Japan \\ ${ }^{3}$ Division of Radiological Technology, Nihon University Hospital, 1-6 Kanda-Surugadai, Chiyoda-ku, Tokyo 101-8309, Japan \\ ${ }^{4}$ Department of Breast Surgery, Nihon University Hospital, 1-6 Kanda-Surugadai, Chiyoda-ku, Tokyo 101-8309, Japan
}

Correspondence should be addressed to Yasuo Amano; yas-amano@nifty.com

Received 17 April 2017; Revised 27 May 2017; Accepted 13 June 2017; Published 24 July 2017

Academic Editor: Marco Moschetta

Copyright (C) 2017 Yasuo Amano et al. This is an open access article distributed under the Creative Commons Attribution License, which permits unrestricted use, distribution, and reproduction in any medium, provided the original work is properly cited.

Purpose. The purpose of this study was to determine texture parameters reflecting the background parenchymal enhancement (BPE) of the breast, which were acquired using texture analysis (TA). Methods. We investigated 52 breasts of the 26 subjects who underwent dynamic contrast-enhanced MRI. One experienced reader scored BPE visually (i.e., minimal, mild, moderate, and marked). TA, including 12 texture parameters, was performed to distinguish the BPE scores quantitatively. Relationships between the visual BPE scores and texture parameters were evaluated using analysis of variance and receiver operating characteristic analysis. Results. The variance and skewness of signal intensity were useful for differentiating between moderate and mild or minimal BPE or between mild and minimal BPE, respectively, with the cutoff value of 356.7 for variance and that of 0.21 for skewness. Some TA features could be useful for defining breast lesions from the BPE. Conclusion. TA may be useful for quantifying the BPE of the breast.

\section{Introduction}

Background parenchymal enhancement (BPE) is defined as the initial enhancement of the normal breast tissue in the standardized dynamic contrast-enhanced magnetic resonance imaging (MRI). BPE is categorized as minimal, mild, moderate, and marked according to the Breast Imaging Reporting and Data System (BI-RADS) [1-3]. The factors that influence the degree of BPE are patients' age, vascular supply to the breast tissues, and endogenous hormone $[1,2]$. An increased BPE can affect identification of enhancing breast carcinoma, especially that of ductal carcinoma in situ (DCIS) or that showing nonmass enhancement, and interfere with definition of the margin of breast cancer [3,4]. Recently, there are controversies about the relationship between the degree of BPE and risk for breast cancer [4-7]. Therefore, the accurate assessment of BPE is important for interpreting breast MRI.

BPE is usually scored on a visual inspection $[2,3]$. Although dedicated training on breast MRI interpretation improves interobserver agreement for the assessment of
BPE, agreement is still moderate [8]. Tagliafico et al. [9] have indicated that their quantitative tool improves BPE assessment. Texture analysis (TA) is a statistical method to analyze the textures of medical images, including signal intensity, its spatial variation, and gray level cooccurrence of the images [10-12]. TA has been already applied to dynamic contrast-enhanced MRI of the breast lesions to differentiate between benign and malignant lesions or characterize breast malignancies $[13,14]$. TA is performed not only on dynamic breast MRI but also on MRI of brain tissues, multiple sclerosis, nasopharyngeal carcinoma, and prostate pathologies [10, 15-18]. In addition, TA is applied to CT to evaluate the heterogeneity of renal carcinoma [19]. However, to our knowledge, TA has not been used to quantify BPE on dynamic breast MRI.

Thus, the purpose of this study was to define texture parameters appropriate for evaluating BPE. In particular, we aimed to differentiate between moderate and mild BPE by utilizing TA on the dynamic contrast-enhanced breast MRI. 


\section{Materials and Methods}

2.1. Patients. Mammogram had shown breast mass, microcalcification, or focal asymmetrical density, and thereafter breast MRI was performed to determine the presence and extent of the lesions in our institution. The present study inclusion criteria were as follows: (1) sufficient image quality on the 1st postcontrast images of MRI, (2) presence of fibroglandular tissues in the breast, (3) no history of biopsy or surgery of the breast, (4) no history of hormonal treatment or chemotherapy, (5) description of visual BPE score according to the BI-RADS on site firstly, (6) an independent reader noted below being able to score BPE based on the BIRADS later, and (7) a region of interest (ROI) for TA being able to be placed on the BPE. As a consequence, between May 2016 and November 2016, 26 women who underwent breast MRI for identifying or diagnosing breast lesions and whose BPE was scored according to the BI-RADS were included in this retrospective study. The median age was 45.5 years (range, $25-80$ years). Histological diagnosis was acquired from 14 of the 26 patients (invasive ductal carcinoma (IDC), 4; fibroadenoma, 4; mastopathy, 4; DCIS, 2). Breast MRI showed no lesions in 10 patients and no histological confirmation was acquired in the remaining 2 patients whose breast MRI had given category $4 \mathrm{~b}$ for the lesions. The study was approved by the institutional ethics committee, and all patients gave informed consent for dynamic contrastenhanced breast MRI.

2.2. MRI Protocol. All MRI studies were performed using a 1.5 T unit (Achieva, Philips Healthcare, Best, the Netherlands). A phased-array coil was used for signal reception. After T1- and fat-suppressed T2-weighted imaging and diffusion-weighted imaging, transverse dynamic contrastenhanced MRI was acquired using fat-suppressed 3-dimensional T1-weighted gradient-echo imaging with the following imaging parameters: repetition time: $4.7 \mathrm{~ms}$; echo time: $2.3 \mathrm{~ms}$; flip angle: $10^{\circ}$; in-plane resolution: $1.0 \times 1.1 \mathrm{~mm}^{2}$; and slice thickness: $2 \mathrm{~mm}$ before zero-filling interpolation. A $k$ space segmentation of 26 and sequential phase ordering were used, and the scan time was $63 \mathrm{sec} /$ phase. The images were acquired before contrast, and $1 \mathrm{~min}, 2 \mathrm{~min}$, and $5 \mathrm{~min}$ after the start of $0.1 \mathrm{mmol} / \mathrm{kg}$ gadolinium injection at a rate of $2 \mathrm{ml} / \mathrm{sec}$ for gadoteridol and $1 \mathrm{ml} / \mathrm{sec}$ for gadobutrol.

2.3. BPE Assessment. BPE was scored on the 1st postcontrast images by an independent investigator with 25 years of experience in diagnostic radiology, including 12 years of experience in breast MRI. The investigator belonged to an institution different from that performing breast MRI and was blinded to clinical information of all patients.

2.4. TA for BPE. The other investigator, who was blinded to the BPE scores, performed TA for the breast parenchyma using MaZda software (version 4.5, Institute of Electronics, Technical University of Lodz, Poland) [10-12]. This is an open-access software for TA developed by the Polish institution, which provides a large amount of texture parameters, including the histogram, gradient, and cooccurrence matrix, which can be related to the medical images placed on the ROI [11]. In the present study, a ROI was placed on the $\mathrm{BPE}$ at the slice including the largest breast parenchyma. We acquired 12 texture parameters as follows: the mean, variance, skewness, and kurtosis of signal intensity (SI), the mean, variance, skewness, and kurtosis of gradient, and entropy in the 4 directions (i.e., EnLL, EnLH, EnHL, and EnHH).

2.5. Statistical Analysis. Data are presented as mean \pm standard deviation. We evaluated the differences in the 12 texture parameters between the $3 \mathrm{BPE}$ scores (i.e., minimal, mild, and moderate), since no patients showed marked BPE in this study. A factorial analysis of variance test was used for the comparison, followed by the post hoc Bonferroni test. $P<0.05$ was defined as statistically significant. A receiver operating characteristic analysis was used to define the cutoff values of texture parameters to differentiate the moderate and mild BPE or between the mild and minimal BPE. When breast lesions were found, we evaluated their texture parameters.

\section{Results}

Among 52 breasts of the 26 patients, the 36, 11, and 6 breasts showed minimal, mild BPE, and moderate BPE, respectively. There were no patients with marked BPE, possibly because the MRI was performed during the menstrual cycle when BPE might be reduced and because of some of patients' age (i.e., menopause).

Table 1 summarizes the TA data of the BPE. There were significant differences in the variance and skewness of SI, mean and variance of gradient, EnLH, and EnHL between the moderate, mild, and minimal BPE $(P<0.01$ for all). The variance of SI was significantly greater in the moderate BPE than in the mild and minimal BPE ( $P<0.01$ for both). The variance of gradient and EnLH were also significantly greater in the moderate BPE than in the mild $(P<0.05$ for both) and minimal BPE $(P<0.01$ for both). The skewness of SI was significantly greater in the moderate and mild BPE than in the minimal BPE ( $P<0.01$ for both). The mean of gradient and EnHL were significantly greater in the moderate BPE than in the minimal BPE $(P<0.05)$.

The SI variance of 356.7 was the perfect cutoff value with an area under the curve (AUC) of 1.00 for distinguishing between the moderate and mild BPE (Figures 1 and 2). The cutoff value and AUC for distinguishing between the moderate and mild BPE were 202.8 and 0.82 for the variance of gradient, respectively, and they were 22.8 and 0.79 for the EnLH, respectively. For differentiating between the mild and minimal BPE, the skewness of SI was only a significant texture parameter, and the cutoff value and AUC were 0.21 and 0.73 , respectively (Figure 3 ).

Among the 14 breast lesions proven histologically, the 6 lesions, including 2 DCIS, 2 IDC, and 2 fibroadenoma, were identified at the slices where TA was analyzed (Figures 1 and 4). The 2 IDC and 1 DCIS showed the variance of SI, mean and variance of gradient, EnLL, EnLH, and EnHL higher than their highest values of BPE (Table 2). The 2 fibroadenoma 
TABLE 1: Texture analysis of background parenchymal enhancement of the breast.

\begin{tabular}{lcccc}
\hline BPE & Minimal $(n=35)$ & Mild $(n=11)$ & Moderate $(n=6)$ & \\
\hline Texture parameters & & & & \\
Mean of SI & $95.1 \pm 23.6$ & $97.4 \pm 14.8$ & $101.2 \pm 14.0$ & 0.8 \\
Variance of SI & $197.8 \pm 161.7$ & $273.1 \pm 77.3$ & $695.2 \pm 236.8$ & $<.72 \pm 0.47$ \\
Skewness of SI & $-0.0 .83 \pm 0.38$ & $0.33 \pm 0.41$ & $0.71 \pm 1.53$ & $<.01^{*}$ \\
Kurtosis of SI & $0.34 \pm 0.62$ & $0.70 \pm 1.08$ & $18.2 \pm 8.19$ & 0.4 \\
Mean of gradient & $14.9 \pm 4.69$ & $17.3 \pm 2.44$ & $159.7 \pm 65.5$ & $<.0 .01^{* * * *}$ \\
Variance of gradient & $81.1 \pm 55.7$ & $99.7 \pm 23.7$ & $0.99 \pm 0.32$ & $<0.01^{* *}$ \\
Skewness of gradient & $0.87 \pm 0.17$ & $0.91 \pm 0.32$ & $1.22 \pm 1.16$ & 0.48 \\
Kurtosis of gradient & $0.84 \pm 0.56$ & $0.92 \pm 1.08$ & $11326 \pm 2967$ & 0.5 \\
EnLL & $9452 \pm 5199$ & $9775 \pm 2997$ & $17.17 \pm 7.10$ & 0.81 \\
EnLH & $8.27 \pm 4.58$ & $11.46 \pm 3.56$ & $24.46 \pm 7.85$ & $<0.01^{* *}$ \\
EnHL & $14.77 \pm 10.68$ & $16.76 \pm 4.73$ & $1.62 \pm 0.55$ & $<0.05^{* * * *}$ \\
EnHH & $2.25 \pm 6.77$ & $1.31 \pm 0.32$ & 0.88 \\
\hline
\end{tabular}

BPE: background parenchymal enhancement, SI: signal intensity, and En: entropy; ${ }^{*}$ the variance of SI was significantly greater in the moderate BPE than in the mild and minimal BPE $(P<0.01$ for both $) .{ }^{* *}$ The variance of gradient and EnLH were also significantly greater in the moderate BPE than in the mild $(P<0.05$ for both $)$ and minimal BPE $\left(P<0.01\right.$ for both). ${ }^{* * *}$ The skewness of SI was significantly greater in the moderate and mild BPE than in the minimal BPE $\left(P<0.01\right.$ for both). ${ }^{* * * *}$ The mean of gradient and EnHL were significantly greater in the moderate BPE than in the minimal BPE $(P<0.05)$.

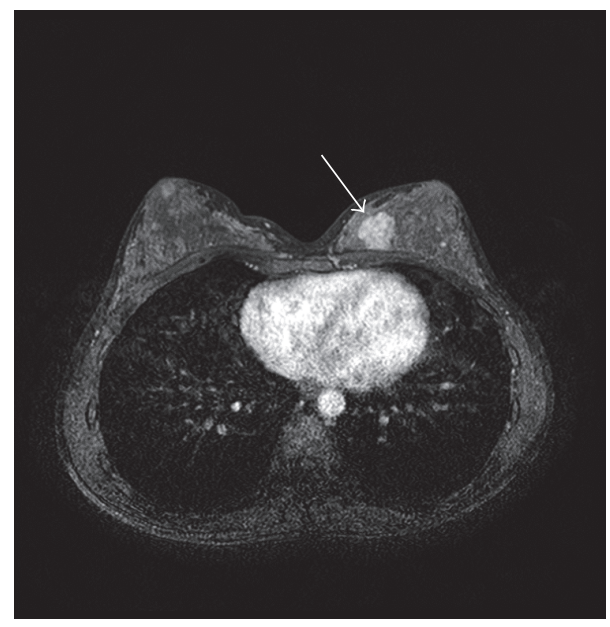

FIGURE 1: A 32-year-old woman with fibroadenoma. Background parenchymal enhancement (BPE) of the right breast is scored as moderate, while that of the left breast is scored as mild. Texture analysis (TA) reveals that the variance of signal intensity (SI) is 356.7 for the right breast and 310.5 for the left breast. A fibroadenoma is hyperintense to BPE (arrows).

showed the mean SI higher than the highest value of BPE (Table 2). One DCIS was recognized visually, but the texture parameters were within ranges of the parameters of BPE.

\section{Discussion}

TA has been applied to characterize breast lesions and their response to the treatments on dynamic contrast-enhanced MRI $[13,14,20,21]$, whereas TA has not previously been used to quantify BPE. The present study demonstrated that the SI variance acquired by TA provided the perfect cutoff value

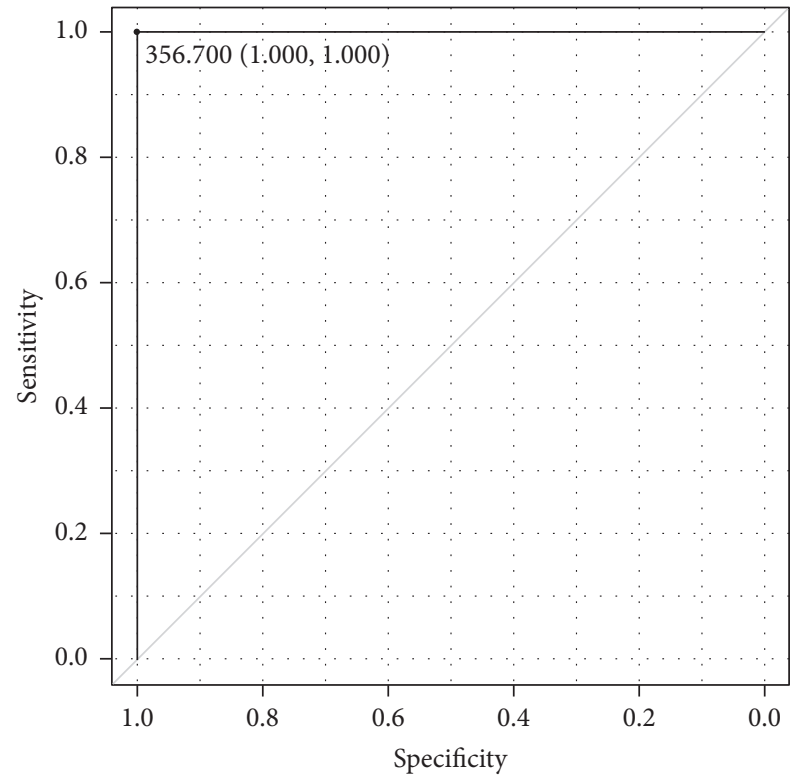

FIGURE 2: The receiver operating characteristic curve shows that the signal intensity variance of 356.7 is the perfect cutoff value between moderate and mild background parenchymal enhancement.

of 356.7 for distinguishing between the moderate and mild $\mathrm{BPE}$ and that the SI skewness was a significant parameter for differentiating between the mild and minimal BPE. Some texture parameters, including the SI variance and EnLL, were higher in 3 of the 4 malignant tumors (i.e., 2 IDC and 1 DCIS) than in BPE. Therefore, the SI variance may be the most useful texture parameter for differentiating between moderate and mild or minimal BPE and between BPE and some malignant lesions. 


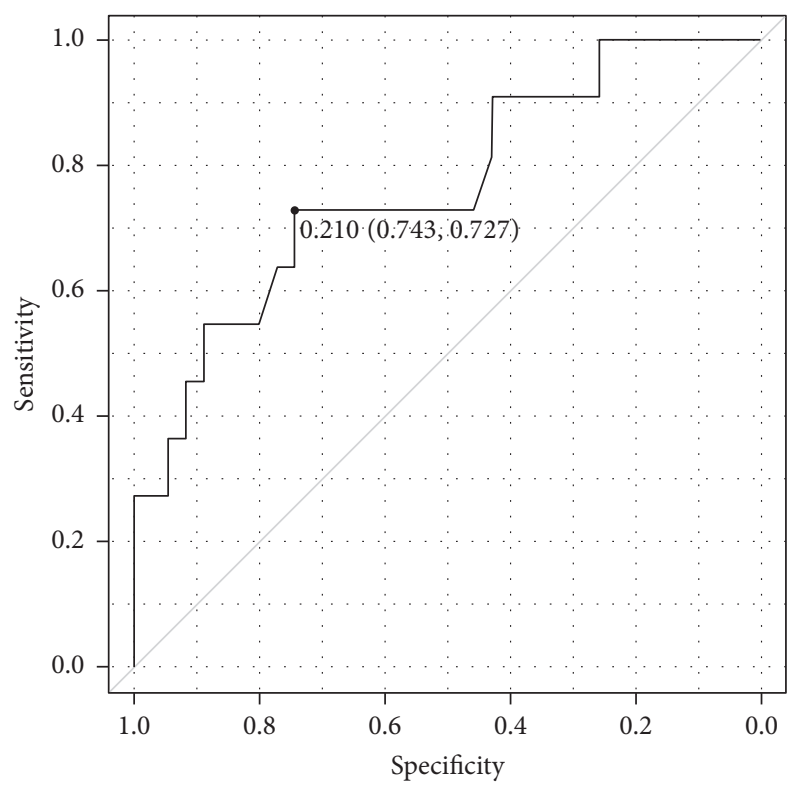

FIGURE 3: The receiver operating characteristic curve shows that skewness of signal intensity distinguishes between mild and minimal background parenchymal enhancement with the cutoff value of 0.21 and area under the curve of 0.73 .

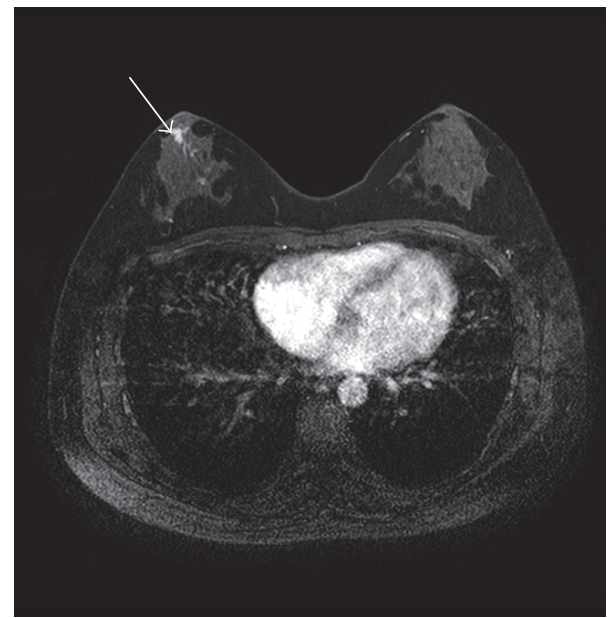

FIGURE 4: A 41-year-old woman with ductal carcinoma in situ. Background parenchymal enhancement (BPE) is scored as minimal. The tumor (arrow) shows the variance of signal intensity, mean and variance of gradient, and entropy LH and HL higher than their highest values of BPE.

Strong BPE can interfere with the identification of DCIS $[3,4]$, and marked or moderate BPE may be one of the risk factors for breast cancer $[5,6]$. Thus, quantitative assessment of BPE is required to support the visual scoring of BPE. In the present study using TA, the variance of SI, variance of gradient, and EnLH were significant discriminators between the moderate BPE and the mild or minimal BPE. Among these parameters, the SI variance provided the perfect cutoff value for distinguishing between them. The variance of SI
TABLE 2: Texture parameters of the 6 breast lesions.

\begin{tabular}{lccccc}
\hline & $\begin{array}{c}\text { Variance } \\
\text { of SI }\end{array}$ & $\begin{array}{c}\text { Mean of } \\
\text { gradient }\end{array}$ & $\begin{array}{c}\text { Variance } \\
\text { of } \\
\text { gradient }\end{array}$ & EnLH & EnHL \\
\hline IDC $^{*}$ & 1382.5 & 35.4 & 353.5 & 60.9 & 52.1 \\
IDC $^{*}$ & 1656.2 & 33.8 & 372.3 & 44.9 & 51.4 \\
DCIS $^{*}$ & 1336.4 & 33.8 & 451 & 45.5 & 77.1 \\
DCIS & 290 & 18.8 & 112.1 & 12.9 & 15 \\
Fibroadenoma & 224.3 & 16.7 & 116.8 & 16.8 & 26.6 \\
Fibroadenoma & 305.8 & 18.9 & 170.7 & 13.9 & 20.6 \\
Highest value of & 685.4 & 26.3 & 248.4 & 22.8 & 45.7 \\
BPE & & & & & \\
\hline
\end{tabular}

SI: signal intensity, En: entropy, IDC: invasive ductal carcinoma, DCIS: ductal carcinoma in situ, and BPE: background parenchymal enhancement. ${ }^{*}$ The 2 IDC and 1 DCIS showed the variance of SI, mean and variance of gradient, EnLH, and EnHL higher than their highest values of BPE.

reflects the change ratios of SI between neighbor pixels or within the tissues. Therefore, our results indicate that the visual scoring of BPE can be affected by the heterogeneity and distribution of BPE. The present study also indicates that the SI variance is a useful parameter for identifying IDC [13]. The relationship between entropy and heterogeneity of the breast cancer is likely different between previous studies $[20,21]$. The use of subtraction images, TA analysis software, or pathological features of the tumors can affect the values of texture parameters. In the present study, only a skewness of SI was a significant discriminator between the mild and minimal BPE. However, the clinical values of differentiating between them may be small because they are not related to the risks for breast cancer $[5,6]$.

Previous studies have shown that the semiautomated or automated assessment of BPE correlates with the radiologists' assessment of BPE and can support their visual inspection [9, 22]. The software developed by some authors has been applied to T1- and T2-weighted imaging as well as dynamic contrastenhanced MRI, but this is home-made [21, 22]. Conversely, the MaZda software used herein is open-access [10-12]. The present study indicates that TA can be applied widely to quantify BPE.

There are several limitations to this study. First, the study population was small. There were a limited number of patients with DCIS. Further study will be requested to determine the usefulness of TA for detecting DCIS surrounded by the moderate BPE. Second, we did not analyze all parameters that could be acquired using TA, because the data handling may be too much to be used clinically. Nonetheless, some texture parameters, including the variance of SI, variance of gradient, and EnLH, were significant discriminators between the moderate BPE and the mild or minimal BPE. Third, TA was performed on only one slice including the largest breast parenchyma, while 3D dynamic contrast-enhanced MRI covered the whole breast. We need a more sophisticated method that can analyze a large amount of TA data rapidly. Fourth, the reference standard was still the BPE scored by an experienced reader visually $[8,9]$. Lastly, neither cutoff value 
nor AUC can be extrapolated to other institutions using 3.0 T or different imaging parameters of the dynamic MRI.

\section{Conclusions}

The variance of SI acquired by TA provided the perfect cutoff value of 356.7 for distinguishing between the moderate and mild BPE in this study. The variance of gradient and EnLH were also significantly greater in the moderate BPE than in the mild BPE. TA can quantify the BPE and help its visual scoring in the clinical practice.

\section{Abbreviations \\ BPE: Background parenchymal enhancement \\ DCIS: Ductal carcinoma in situ \\ En: Entropy \\ ROI: Region of interest \\ TA: Texture analysis.}

\section{Conflicts of Interest}

All authors had no conflicts of interest.

\section{Authors' Contributions}

Yasuo Amano designed the research, analyzed the TA, and drafted the paper. Jun Woo, Hiroshi Yamamoto, and Fumi Yanagisawa examined breast MRI. Maki Amano scored the BPE of all cases in a blinded manner. Mayumi Tani enrolled the patients for this study and performed the biopsy of some lesions.

\section{References}

[1] C. K. Kuhl, H. B. Bieling, J. Gieseke et al., "Healthy premenopausal breast parenchyma in dynamic contrast-enhanced MR imaging of the breast: Normal contrast medium enhancement and cyclical-phase dependency," Radiology, vol. 203, no. 1, pp. 137-144, 1997.

[2] E. A. Morris, C. Comstock, C. D. Lehman et al., B. Back Ground Parenchymal Enhancement (BPE), ACR BI-RADS ${ }^{\circledR}$ Magnetic Resonance Imaging, ACR BI-RADS ${ }^{\circledR}$ Atlas, 5th edition, 2013.

[3] C. S. Giess, E. D. Yeh, S. Raza, and R. L. Birdwell, "Background parenchymal enhancement at breast MR imaging: Normal patterns, diagnostic challenges, and potential for false-positive and false-negative interpretation," Radiographics, vol. 34, no. 1, pp. 234-247, 2014.

[4] S. Y. Park, D. K. Kang, and T. H. Kim, "Does background parenchymal enhancement on MRI affect the rate of positive resectionmargin in breast cancer patients?” British Journal of Radiology, vol. 88, no. 1046, Article ID 20140638, 2015.

[5] V. King, J. D. Brooks, J. L. Bernstein, A. S. Reiner, M. C. Pike, and E. A. Morris, "Background parenchymal enhancement at breast MR imaging and breast cancer risk," Radiology, vol. 260, no. 1, pp. 50-60, 2011.

[6] M. Telegrafo, L. Rella, A. A. Stabile Ianora, G. Angelelli, and M. Moschetta, "Breast MRI background parenchymal enhancement (BPE) correlates with the risk of breast cancer," Magnetic Resonance Imaging, vol. 34, no. 2, pp. 173-176, 2016.
[7] B. Bennani-Baiti, M. Dietzel, and P. A. Baltzer, "MRI background parenchymal enhancement is not associated with breast cancer," PLoS ONE, vol. 11, no. 7, Article ID e0158573, 2016.

[8] A. Melsaether, M. McDermott, D. Gupta, K. Pysarenko, S. D. Shaylor, and L. Moy, "Inter- And intrareader agreement for categorization of background parenchymal enhancement at baseline and after training," American Journal of Roentgenology, vol. 203, no. 1, pp. 209-215, 2014.

[9] A. Tagliafico, B. Bignotti, G. Tagliafico, S. Tosto, A. Signori, and M. Calabrese, "Quantitative evaluation of background parenchymal enhancement (BPE) on breast MRI. A feasibility study with a semi-automatic and automatic software compared to observer-based scores," British Journal of Radiology, vol. 88, no. 1056, Article ID 20150417, 2015.

[10] G. Castellano, L. Bonilha, L. M. Li, and F. Cendes, "Texture analysis of medical images," Clinical Radiology, vol. 59, no. 12, pp. 1061-1069, 2004.

[11] A. Materka, "Texture analysis methodologies for magnetic resonance imaging," Dialogues in Clinical Neuroscience, vol. 6, no. 2, pp. 243-250, 2004.

[12] P. M. Szczypiński, M. Strzelecki, A. Materka, and A. Klepaczko, "MaZda-a software package for image texture analysis," Computer Methods and Programs in Biomedicine, vol. 94, no. 1, pp. 66-76, 2009.

[13] W. Chen, M. L. Giger, H. Li, U. Bick, and G. M. Newstead, "Volumetric texture analysis of breast lesions on contrastenhanced magnetic resonance images," Magnetic Resonance in Medicine, vol. 58, no. 3, pp. 562-571, 2007.

[14] S. A. Waugh, C. A. Purdie, L. B. Jordan et al., "Magnetic resonance imaging texture analysis classification of primary breast cancer," European Radiology, vol. 26, no. 2, pp. 322-330, 2016.

[15] M. E. Mayerhoefer, G. H. Welsch, G. Riegler et al., "Feasibility of texture analysis for the assessment of biochemical changes in meniscal tissue on $\mathrm{tl}$ maps calculated from delayed gadoliniumenhanced magnetic resonance imaging of cartilage data: Comparison with conventional relaxation time measurements," Investigative Radiology, vol. 45, no. 9, pp. 543-547, 2010.

[16] J. Liu, Y. Mao, Z. Li et al., "Use of texture analysis based on contrast-enhanced MRI to predict treatment response to chemoradiotherapy in nasopharyngeal carcinoma," Journal of Magnetic Resonance Imaging, vol. 44, no. 2, pp. 445-455, 2016.

[17] R. Rozenberg, R. E. Thornhill, T. A. Flood, S. W. Hakim, C. Lim, and N. Schieda, "Whole-tumor quantitative apparent diffusion coefficient histogram and texture analysis to predict Gleason score upgrading in intermediate-risk $3+4=7$ prostate cancer," American Journal of Roentgenology, vol. 206, no. 4, pp. 775-782, 2016.

[18] R. K. Verma, J. Slotboom, C. Locher et al., "Characterization of Enhancing MS Lesions by Dynamic Texture Parameter Analysis of Dynamic Susceptibility Perfusion Imaging," BioMed Research International, vol. 2016, Article ID 9578139, 2016.

[19] M. G. Lubner, N. Stabo, E. J. Abel, A. Munoz Del Rio, and P. J. Pickhardt, "CT textural analysis of large primary renal cell carcinomas: Pretreatment tumor heterogeneity correlates with histologic findings and clinical outcomes," American Journal of Roentgenology, vol. 207, no. 1, pp. 96-105, 2016.

[20] J. Parikh, M. Selmi, G. Charles-Edwards et al., "Changes in primary breast cancer heterogeneity may augment midtreatment MR imaging assessment of response to neoadjuvant chemotherapy," Radiology, vol. 272, no. 1, pp. 100-112, 2014. 
[21] J. Kim, E. S. Ko, Y. Lim et al., "Breast cancer heterogeneity: MR imaging texture analysis and survival outcomes," Radiology, vol. 282, no. 3, pp. 665-675, 2017.

[22] A. Tagliafico, B. Bignotti, G. Tagliafico et al., "Breast density assessment using a 3T MRI system: Comparison among different sequences," PLoS ONE, vol. 9, no. 6, Article ID e99027, 2014. 


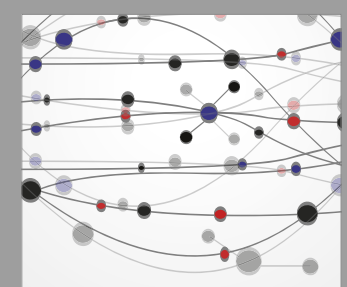

The Scientific World Journal
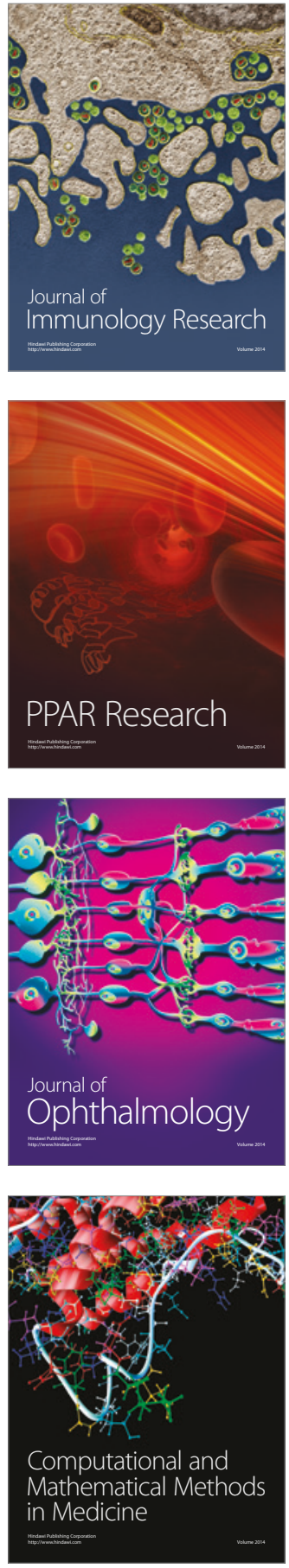

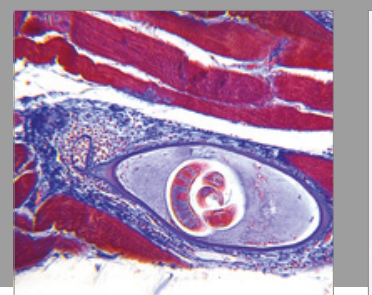

Gastroenterology Research and Practice
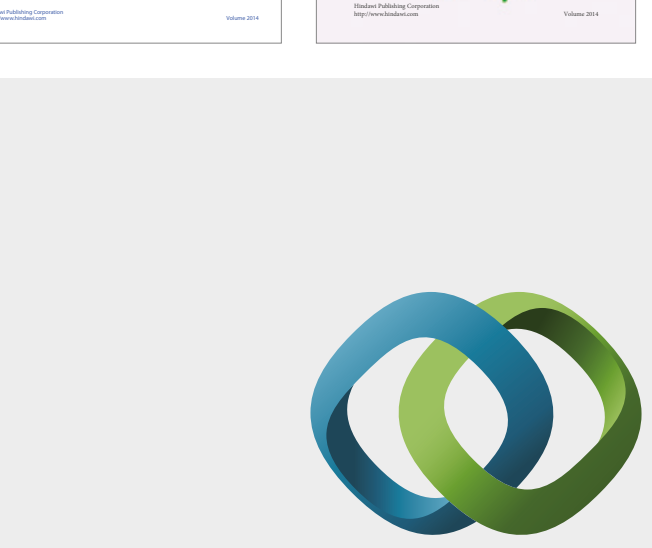

\section{Hindawi}

Submit your manuscripts at

https://www.hindawi.com
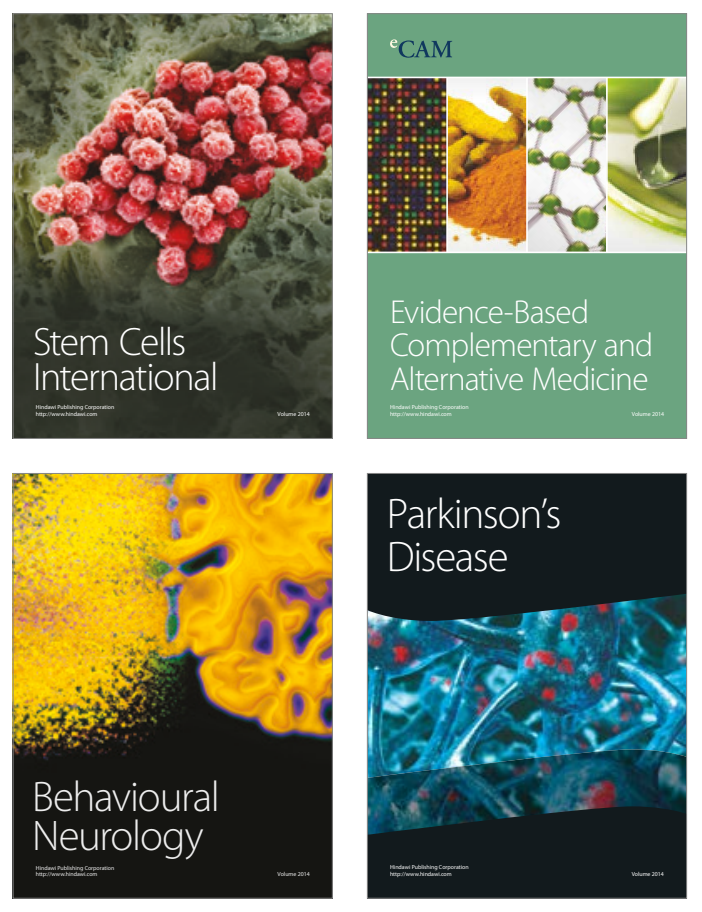
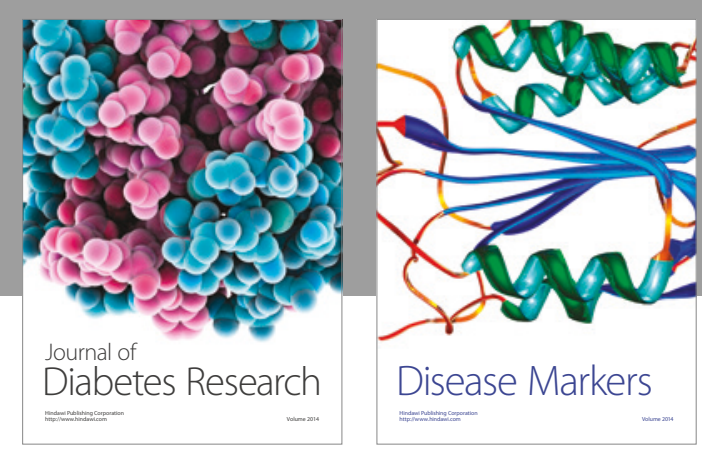

Disease Markers
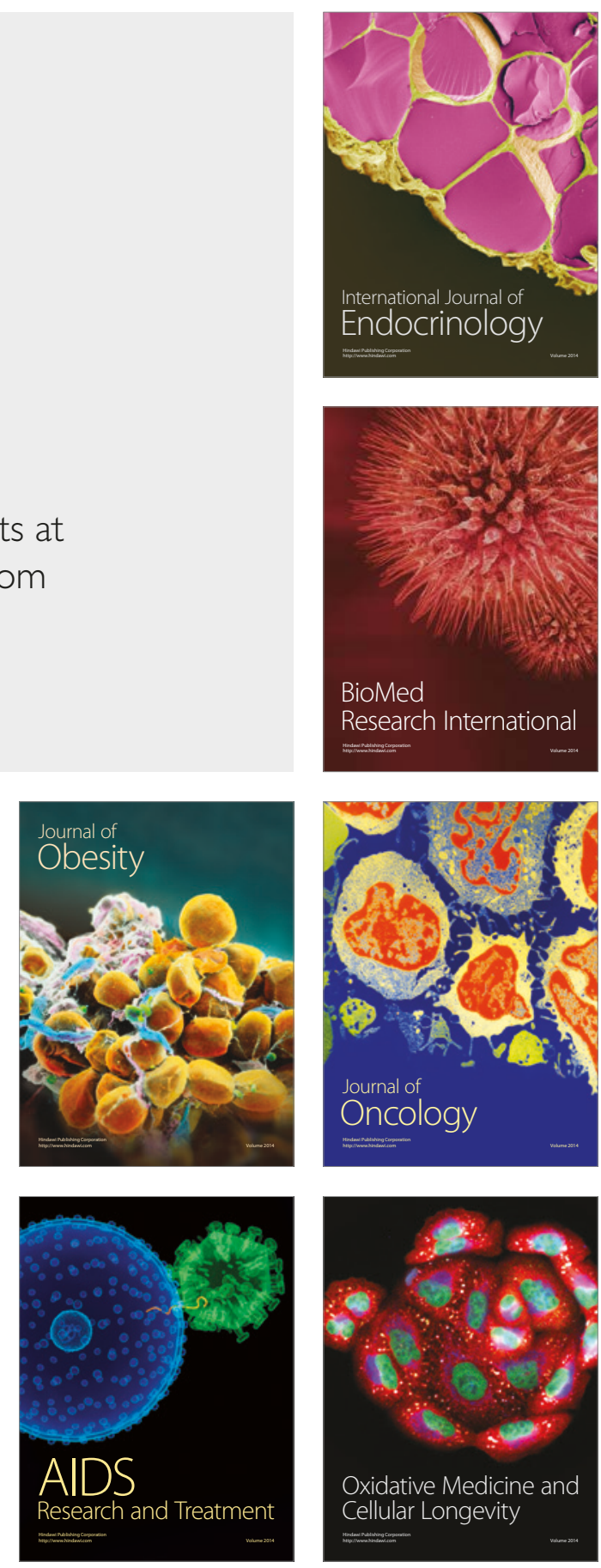